\title{
A TWO-GENERATOR PRESENTATION FOR THE PICARD GROUP
}

\author{
A. M. BRUNNER
}

(Communicated by Warren J. Wong)

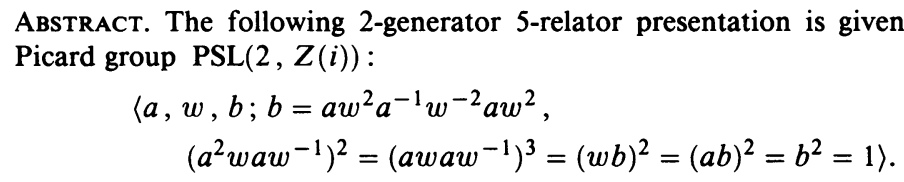

The Picard group, $P=\operatorname{PSL}(2, Z(i))$, has a well-known presentation $P=$ $\left\langle u, x, y, v ; u^{2}=x^{3}=y^{3}=v^{2}=(x v)^{2}=(x y)^{2}=(u y)^{2}=(u v)^{2}=1\right\rangle$ ([2] or [1]). This presentation comes from looking at rotations on the edges of a fundamental polyhedron for $P$ in $\mathbb{H}^{3}$. With the usual convention for matrices in PSL, a faithful representation is given with

$$
u=\left[\begin{array}{ll}
0 & i \\
i & 0
\end{array}\right], \quad x=\left[\begin{array}{ll}
0 & i \\
i & 1
\end{array}\right], \quad y=\left[\begin{array}{cc}
1 & 1 \\
-1 & 0
\end{array}\right], \quad \text { and } \quad v=\left[\begin{array}{cc}
0 & 1 \\
-1 & 0
\end{array}\right] \text {. }
$$

We show below that $P$ is generated by

$$
x u=\left[\begin{array}{cc}
1 & 0 \\
-i & 1
\end{array}\right] \text { and } v u y=\left[\begin{array}{ll}
i & i \\
i & 0
\end{array}\right]
$$

in a presentation with five relators. The reduction to three generators is standard for presentations of this sort; the reduction to two generators is more unexpected.

The first reduction in generators is obtained with $a=x u, b=u y$ so that $x=a u, y=u b$. Thus

$P=\left\langle u, a, b, v ; u^{2}=(a u)^{3}=(u b)^{3}=v^{2}=(a u v)^{2}=(a b)^{2}=b^{2}=(u v)^{2}=1\right\rangle$.

Now, using $(u v)^{2}=(a u)^{3}=u^{2}=v^{2}=1$, we have auvauv = avuauv= $a v a^{-1} u^{-1} a^{-1} v$. It follows that we may replace the relation $(a u v)^{2}=1$ with $u=a^{-1} v a v a^{-1}$. Consequently,

$$
\begin{array}{r}
P=\left\langle a, b, v ;\left(a^{-1} v a v a^{-1}\right)^{2}=\left(v a v a^{-1}\right)^{3}=\left(a^{-1} v a v a^{-1} b\right)^{3}\right. \\
\left.=v^{2}=(a b)^{2}=b^{2}=\left(a^{-1} v a v a^{-1} v\right)^{2}=1\right\rangle .
\end{array}
$$

Moreover, the relation $\left(a^{-1} v a v a^{-1} v\right)^{2}=1$ can be omitted, since from $\left(a^{-1} v_{a v a} a^{-1}\right)^{2}=v^{2}=1,1=v_{a v a}{ }^{-1}$ vava $^{-1}$ vava $^{-1}=v_{\text {avava }}{ }^{-1}$ vavava $^{-1}=$ $\left(v a v a v a^{-1}\right)^{2}=\left(a v a^{-1} v a^{-1} v\right)^{-2}$.

Received by the editors October 25, 1990 and, in revised form, November 30, 1990.

1980 Mathematics Subject Classification (1985 Revision). Primary 20F05. 
For the second reduction, let $w=v b$, so that $v=w b$. Then

$$
\begin{aligned}
P= & \left\langle a, b, w ;\left(a^{-1} w b a w b a^{-1}\right)^{2}=\left(w b a w b a^{-1}\right)^{3}\right. \\
& \left.=\left(a^{-1} w b a w b a^{-1} b\right)^{3}=(w b)^{2}=(a b)^{2}=b^{2}=1\right\rangle .
\end{aligned}
$$

Here $b^{-1} a b=a^{-1}, b^{-1} w b=w^{-1}, b^{2}=1$. The relation $\left(a^{-1} w b a w b a^{-1} b\right)^{3}$ $=1$ can be replaced by $\left(a^{-1} w b a w a\right)^{3}=1$ or $\left(w^{2} b a\right)^{3}=1$. From this last relation, $1=w^{2} b a w^{2} b a w^{2} b a=w^{2} a^{-1} w^{-2} a w^{2} b a$ so that $b=a w^{2} a^{-1} w^{-2} a w^{2}$. Thus $P$ is generated by $a=x u$ and $w=v u y$ as claimed.

Simplifying a little, we obtain

$$
\begin{aligned}
& P=\left\langle a, w, b ; b=a w^{2} a^{-1} w^{-2} a w^{2},\right. \\
& \left.\quad\left(a^{2} w a w^{-1}\right)^{2}=\left(a w a w^{-1}\right)^{3}=(w b)^{2}=(a b)^{2}=b^{2}=1\right\rangle .
\end{aligned}
$$

It is interesting to observe the central role played by the unimodular group $\operatorname{sgp}\left\{a, w a w^{-1}\right\}$. We have not succeeded in finding a more economical presentation for $P$ than this.

\section{REFERENCES}

1. A. M. Brunner, M. L. Frame, Y. W. Lee, and N. J. Wielenberg, Classifying torsion-free subgroups of the Picard group, Trans. Amer. Math. Soc. 282 (1984), 205-235.

2. Benjamin Fine, The HNN and generalized free product structure of certain linear groups, Bull. Amer. Math. Soc. 81 (1975), 413-416.

Department of Mathematics, University of Wisconsin-Parkside, Kenosha, Wisconsin 53141 